OPEN ACCESS

Edited by: Elena Surkova, Royal Brompton Hospital, United Kingdom

Reviewed by: Andrea Agnes Molnar Semmelweis University, Hungary Ali Hosseinsabet,

Tehran University of Medical

Sciences, Iran

*Correspondence:

Yosuke Nabeshima

y.nabeshima1016@gmail.com

Specialty section:

This article was submitted to

Cardiovascular Imaging,

a section of the journal

Frontiers in Cardiovascular Medicine

Received: 14 October 2021 Accepted: 22 November 2021 Published: 13 December 2021

Citation:

Nabeshima Y, Kitano T and Takeuchi M (2021) Prognostic Value of the Three-Dimensional Right Ventricular Ejection Fraction in Patients With Asymptomatic Aortic Stenosis. Front. Cardiovasc. Med. 8:795016. doi: 10.3389/fcrm.2021.795016

\section{Prognostic Value of the} Three-Dimensional Right Ventricular Ejection Fraction in Patients With Asymptomatic Aortic Stenosis

\author{
Yosuke Nabeshima $^{1 *}$, Tetsuji Kitano ${ }^{2}$ and Masaaki Takeuchi ${ }^{3}$ \\ 1 Second Department of Internal Medicine, School of Medicine, University of Occupational and Environmental Health, \\ Kitakyushu, Japan, ${ }^{2}$ Department of Cardiology and Nephrology, Wakamatsu Hospital of University of Occupational and \\ Environmental Health, Kitakyushu, Japan, ${ }^{3}$ Department of Laboratory and Transfusion Medicine, School of Medicine, \\ Hospital of University of Occupational and Environmental Health, Kitakyushu, Japan
}

Background: The right ventricular (RV) function is an important prognostic marker of asymptomatic aortic stenosis (AS). However, previous publications have not addressed the additive value of conventional RV parameters over left heart parameters. Whether three-dimensional echocardiography (3DE)-derived RV ejection fraction (RVEF) has prognostic utility independent of 3DE derived left heart parameters is also unknown. We investigated the prognostic utility of 3DE RVEF in patients with asymptomatic AS.

Methods: We retrospectively selected 392 asymptomatic AS patients. RVEF, left ventricular ejection fraction (LVEF) and left atrial volumes (LAVs) were measured using 3DE datasets. We determined the association of those parameters, as well as of aortic valve replacement (AVR), and Charlson's comorbidity index with cardiac events. We also analyzed whether RVEF has incremental value over two-dimensional echocardiography (2DE) RV parameters.

Results: During a median follow-up of 27 months, 57 patients developed cardiac events, and 68 patients received AVR. Univariate Cox proportional hazard analysis revealed that RVEF was associated with cardiac events $(p<0.001)$. Multivariate analysis revealed that RVEF was significantly associated with cardiac events $(p<0.001)$ even after adjusting for AVR, Charlson's comorbidity index, LVEF, LAV, E/e', and indexed aortic valve area (iAVA). An incremental value of RVEF over left heart parameters was also demonstrated using a nested regression model. Classification and regression-tree analysis selected RVEF first with a cut-off value of $41 \%$. RVEF had incremental value over iAVA, LVEF, and 2DE conventional RV parameters for its association with future outcomes.

Conclusions: 3DE RVEF had significant prognostic value even after adjusting for comorbidities, left heart parameters, and conventional 2DE RV parameters in asymptomatic aortic stenosis.

Keywords: asymptomatic aortic stenosis, prognosis, 3DE, RVEF, CART analysis 


\section{INTRODUCTION}

The number of patients with aortic stenosis (AS) has been increasing rapidly due to the aging of society, especially in developed countries (1). This change has resulted in an exponential increase of heart failure (HF) hospitalizations and surgical or transcatheter aortic valve replacements (AVRs) (2). Due to the socioeconomic impact of AS, optimization of surgical intervention is urgently required. Current guidelines recommend AVR for patients with symptomatic severe AS (3). However, management of asymptomatic AS with preserved left ventricular ejection fraction (LVEF) has not been clarified (36). When considering AVR in asymptomatic AS patients $(6,7)$, we have to assess not only valve condition, but also myocardial function, because of the non-negligible rate of sudden cardiac death and relatively higher risk of HF (8-10). The prognostic impact of right ventricular (RV) function has garnered increased interest in this parameter in recent years. RV dysfunction can affect the risk of HF via a negative impact on cardiac output and ventricular interactions in various heart diseases (11). Genereux and colleagues have proposed a new concept called "cardiac damage stage" for risk stratification of asymptomatic AS patients $(12,13)$. According to their findings, patients with RV dysfunction had worse prognoses than those with left ventricular (LV) dysfunction with preserved RV function. Galli et al. also indicated the importance of tricuspid annular plane systolic excursion (TAPSE) to predict cardiovascular death in severe AS (14). However, those publications used 2D or Doppler echocardiography to analyze RV function, irrespective of complex RV geometry. Three-dimensional echocardiography (3DE) does not rely on geometric assumptions and can provide more accurate and reliable information regarding RV function (15). Left atrial (LA) function is also reported as a useful marker to stratify the risk of asymptomatic AS patients (16). We hypothesized that RV ejection fraction (RVEF) is the most robust predictor for future outcomes among LV, LA, and RV function parameters assessed with $3 \mathrm{DE}$ in asymptomatic AS. Accordingly, we sought to investigate the prognostic utility of $3 \mathrm{DE}$-derived RVEF in patients with asymptomatic AS.

\section{MATERIALS AND METHODS}

\section{Study Population}

This was a single-center observational study. Using a $3 \mathrm{DE}$ database, we retrospectively selected $3 \mathrm{DE}$ datasets of Japanese patients with AS who had undergone transthoracic echocardiography in the University of Occupational and Environmental Health hospital from April 2008 to December 2018. Individuals who had no AS-related symptoms were selected from the database. Patients with fewer than 30 days of follow-up after echocardiography were excluded. We also collected several clinical parameters to calculate Charlson's comorbidity index (17). The study was approved by the ethics committee at the University of Occupational and Environmental Health. As this was a retrospective study, the Institutional Review Board waved the requirement for informed consent.

\section{Echocardiography}

3DE was performed immediately after standard transthoracic 2D echocardiography (2DE) and Doppler echocardiography. $3 \mathrm{DE}$ images were acquired using an apical approach and commercially available ultrasound machines (iE33 or Epic7G, Philips Healthcare, Andover, MA; Vivid7 or Vivid E95, GE Healthcare, Horten, Norway) equipped with a 3DE transducer (X3-1 or X5-1, Philips Healthcare, Andover, MA; 4V, GE Healthcare, Horten, Norway). Trans-mitral flow velocity was recorded at the coaptation point of both leaflets. Mitral annular velocities were recorded at septal and lateral sides of the mitral annulus, and average e' was calculated. Peak aortic flow velocity was recorded in multiple transducer positions, and the highest value was used for the measurements of the mean pressure gradient (PG) and velocity-time integral (VTI). RV fractional area change (RVFAC) was calculated by standard formula. 2DE RV speckle tracking analysis was performed using commercially available, vendor-independent, fully automated strain analysis software (AutoStrain RV, TomTec Imaging Systems, Unterschleissheim, Germany). The software automatically determined the RV endocardial border and performed speckle tracking analysis during a single cardiac cycle. The endocardial border was manually corrected as required. RV free-wall longitudinal strain (RVfwLS) and global longitudinal strain (RVGLS) were calculated.

\section{D Speckle Tracking Analysis}

LV and RV 3DE speckle tracking analyses were performed using commercially available, vendor-independent, 3DE quantification software (4D LV ANALYSIS-3 and 4D RV FUNCTION 3, TomTec Imaging Systems). These methods have been described in detail previously, and their accuracy and reliability are recognized (18). For LV analysis, after selecting a specific 3DE dataset, the software automatically detected the LV endocardial border. Manual correction of the endocardial border was performed as needed. After confirming the tracing line, 3D speckle-tracking analysis was performed through a single cardiac cycle, generating $\mathrm{LV}$ volume curves, from which $\mathrm{LV}$ end-diastolic volume (LVEDV), end-systolic volume (LVESV), and LVEF were calculated. LV mass was calculated by manually drawing the epicardium of end-diastolic frames of three standard apical views extracted from 3DE datasets. LVEDV index (LVEDVI), LVESV index (LVESVI), and LV mass index (LVMI) were calculated by body surface area (BSA). For the RV analysis, LV-focused end-diastolic apical four-, two-, and three-chamber views, and RV-focused two-orthogonal views were extracted from a 3DE dataset. After specifying anatomical landmarks (center points of the mitral and tricuspid annular planes and the apex), the software automatically determined the RV endocardial cast. Following manual correction of the endocardial border, 3D speckle tracking analysis was performed. RV end-diastolic volume index (RVEDVI), end-systolic volume index (RVESVI), and RVEF were calculated (Figure 1).

\section{D Left Atrial Volume}

3D maximal and minimal left atrial volumes (LAVs) were calculated using vendor-specific software (QLAB 13.0, Philips 


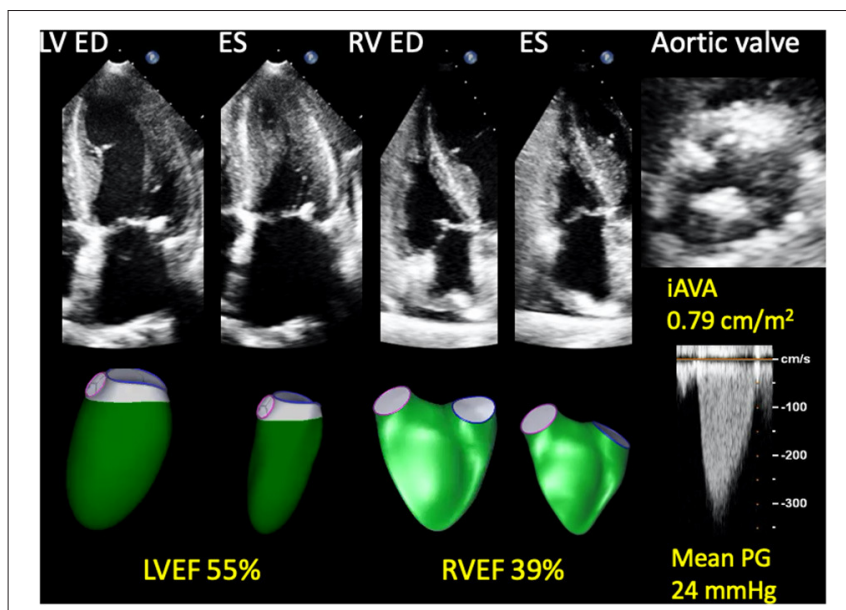

FIGURE 1 | Representative 3D RV and LV analyses in a patient with asymptomatic AS who developed a cardiac event. A 74-year-old woman who developed heart failure three months after baseline echocardiography. AS was moderate and LVEF was preserved; however, RVEF was reduced. She also had hypertension, chronic kidney disease, and chronic obstructive pulmonary disease. 3D, three-dimensional; AS, aortic stenosis; ED, end-diastole; ES, end-systole; iAVA, indexed aortic valve area; LV, left ventricular; LVEF, left ventricular ejection fraction; PG, pressure gradient; RV, right ventricular; RVEF, right ventricular ejection fraction.

Healthcare, Andover, MA; EchoPAC version 203, GE Healthcare, Horten, Norway). Detailed methods have been described previously (19). Briefly, we calculated LAVs using Simpson's biplane method with the anterior-posterior and medial-lateral 2D views extracted from $3 \mathrm{DE}$ datasets. LAVs were indexed to BSA yielding maximal LAV index (LAVIx) and minimal LAV index (LAVIn). This is a manual method, and we did not use 3D LA speckle tracking software.

\section{AS Severity}

Stroke volume (SV) was calculated as the difference of LVEDV and LVESV measured by 3DE. Stroke volume index (SVi) was calculated as SV divided by BSA. Indexed aortic valve area (iAVA) was calculated as SV divided by AV VTI times BSA. We defined more-than-moderate-to-severe AS as AVA $<1.0 \mathrm{~cm}^{2}$ or iAVA $<0.6 \mathrm{~cm}^{2} / \mathrm{m}^{2}$. The patients were classified as high-gradient (peak velocity $\geq 4.0 \mathrm{~m} / \mathrm{s}$ or mean $\mathrm{PG} \geq 40 \mathrm{mmHg}$ ) AS (HGAS), and low-gradient (peak velocity $<4.0 \mathrm{~m} / \mathrm{s}$ and mean PG $<40 \mathrm{mmHg}$ ) AS (LG-AS) according to the current ESC guideline (20). LG-AS was further classified into 3 categories: low-flow (SVi $<35 \mathrm{~mL} / \mathrm{m}^{2}$ ) low-gradient AS (LFLG-AS) with preserved LVEF, LFLG-AS with reduced LVEF, and normal-flow low-gradient AS (NFLG-AS).

\section{Follow-Up}

Patients were followed up in an outpatient clinic. Prognostic information was obtained by interviewing attending physicians or by searching digital medical records. If patients were followed up in other hospitals, we also contacted corresponding physicians to obtain prognostic information. The primary endpoint was defined as a composite adverse cardiac event, including
TABLE 1 | Patient characteristics $(n=367)$.

\begin{tabular}{|c|c|}
\hline \multicolumn{2}{|l|}{ Variables } \\
\hline Age (y.o) & $77.1 \pm 10.0$ \\
\hline Sex (Male) & $168(46 \%)$ \\
\hline Body mass index $\left(\mathrm{kg} / \mathrm{m}^{2}\right)$ & $22.7 \pm 3.9$ \\
\hline Body surface area $\left(\mathrm{m}^{2}\right)$ & $1.52 \pm 0.21$ \\
\hline Systolic blood pressure $(\mathrm{mmHg})$ & $147.5 \pm 23.5$ \\
\hline Diastolic blood pressure $(\mathrm{mmHg})$ & $75.0 \pm 13.5$ \\
\hline Heat Rate (bpm) & $69.5 \pm 12.6$ \\
\hline Rhythm (SR/Af/PM) & $338 / 25 / 4$ \\
\hline \multicolumn{2}{|l|}{ Comorbidities } \\
\hline Hypertension & $295(80 \%)$ \\
\hline Diabetes mellitus & 115 (31\%) \\
\hline Coronary artery disease & $79(22 \%)$ \\
\hline Chronic kidney disease & $174(47 \%)$ \\
\hline Chronic pulmonary disease & $62(17 \%)$ \\
\hline \multicolumn{2}{|l|}{ Medications } \\
\hline Calcium channel blocker & $185(52 \%)$ \\
\hline$\beta$-blocker & $76(21 \%)$ \\
\hline ACEi or ARB & 208 (58\%) \\
\hline Digitalis & $7(2 \%)$ \\
\hline Diuretics & $107(30 \%)$ \\
\hline Warfarin/DOAC & $58(16 \%)$ \\
\hline Charlson's index & $5.22 \pm 1.94$ \\
\hline
\end{tabular}

Data are shown as mean \pm standard deviations or $n(\%)$.

$A C E i$, angiotensin converting enzyme inhibitor; Af, atrial fibrillation; $A R B$, angiotensin receptor blocker; DOAC, direct oral anticoagulant; SR, sinus rhythm; PM, pacemaker.

cardiac death, hospitalization due to heart failure, ventricular tachycardia/fibrillation, or non-fatal myocardial infarction.

\section{Statistical Analysis}

Continuous data were expressed as means \pm SDs or as medians and interquartile ranges (IQR; 25 th to 75 th percentiles). Categorical variables were presented as absolute numbers or percentages. Student's $t$-test was used to compare continuous variables between two groups when data were normally distributed. Wilcoxon sum rank test was used when data were not normally distributed. Fisher's exact test or a chisquare test were used to compare categorical variables. Cox proportional hazards analysis was used to calculate hazard ratios and $95 \%$ confidence intervals. Univariate and multivariate Cox proportional hazard analyses were used to assess the prognostic utility of echocardiographic parameters. AVR was treated as a time-dependent covariate. Survival analysis was performed using the Kaplan-Meier method, and differences in survival rates between groups were analyzed by the log-rank test. Netreclassification improvement (NRI) analysis and DeLong's test were used to compare risk prediction utility between the two models. Classification and regression-tree (CART) analyses were conducted to predict cardiac events. A two-sided $p$-value $<0.05$ was considered statistically significant. All statistical analyses were performed using commercial software (JMP version 14.0, SAS Institute Inc., Cary, NC; R version 3.6.3, The R foundation for Statistical Computing, Vienna). 


\section{RESULTS}

\section{Patient Characteristics}

Using a 3DE database, we found 392 asymptomatic AS patients. Of those patients, 25 were excluded because their follow-up duration after baseline echocardiography was $<30$ days. Finally, first-time echocardiographic datasets from 367 patients were selected for the analysis. We could not analyze 3DE datasets of twelve patients due to a low volume rate or extremely poor image quality. We performed 3DE LV and RV analysis in the other 355 patients, resulting in feasibility of $97 \%$ for both LV and RV analyses. Image quality of LV was good in 35\%, fair in 53\%, and poor in $12 \%$. Corresponding RV values were good in $13 \%$, fair in $57 \%$, and poor in $30 \%$. It was impossible to analyze $3 \mathrm{D}$ LAVs due to the fact that datasets failed to encompass all of the LA wall in 10 patients, and the feasibility of 3D LA analysis was 94\%. LA image quality was good in $35 \%$, fair in $53 \%$, and poor in $11 \%$. Clinical characteristics are summarized in Table $\mathbf{1 .}$

\section{Two- and Three-Dimensional Echocardiographic Parameters}

Severe AS was observed in $43 \%(152 / 354)$ of the study population. Median values of LVEF and RVEF by $3 \mathrm{DE}$ analysis were 52 and $48 \%$, respectively. $30 \%$ (107/355) of patients had reduced LVEF (LVEF <50\%), and 32\% (112/355) had reduced RVEF (RVEF <45\%). 53\% (189/355) of patients had preserved both ventricular EFs, and $15 \%$ (53/355) of patients had reduced bilateral ventricular EFs. Table 2 presents echocardiography parameters.

\section{Outcomes}

During a median follow-up of 26.7 months (IQR, 15.4-56.6 months), 57 patients reached a primary endpoint, including 19 cardiac deaths, $32 \mathrm{HFs}$ requiring hospital admission, four myocardial infarctions, and two ventricular tachyarrhythmias. Notably, four cardiac deaths and three HFs occurred after AVR. Sixty-eight patients received AVR during the follow-up. The median interval from baseline echocardiography to AVR was 20.5 months. Univariate Cox proportional hazard analysis revealed that mean PG, iAVA, E/e', SVi, Charlson's comorbidity index, LVEF, RVEF, and LAVIs were associated with cardiac events (Table 3).

Table 4 depicts the results of multivariate Cox proportional hazard analyses. According to results from univariate analysis and clinical importance, we created five models for multivariate analyses. LVEF and RVEF were significantly associated with cardiac events after adjusting Charlson's comorbidity index, AVR, and one of either echocardiographic parameter. DeLong's test revealed a significant increase of c-statistics after adding RVEF to LVEF, LAVIn, E/e, and mean PG (Figure 2). NRI analysis revealed that the logistic regression model that included RVEF showed a significant improvement of outcome classification compared with the logistic regression model including LVEF, LAVIn, E/e', and mean PG (NRI = 0.655; $p<$ 0.001).

For survival CART analysis, we entered 18 variables including age, sex, Charlson's comorbidity index, LVEDVI, LVESVI, LVEF,
TABLE 2 | Echocardiographic parameters $(n=367)$.

\begin{tabular}{|c|c|c|}
\hline Variables & Number & Median (25th to 75th percentiles) \\
\hline E-wave $(\mathrm{cm} / \mathrm{s})$ & 367 & $77.0(62.0-98.0)$ \\
\hline$E / A$ & 335 & $0.71(0.59-0.90)$ \\
\hline$E / e^{\prime}$ & 363 & $16.9(12.8-22.1)$ \\
\hline MR (severe/moderate/mild) & 318 & $3 / 5 / 219$ \\
\hline TR (severe/moderate/mild) & 274 & $3 / 12 / 218$ \\
\hline SPAP (mmHg) & 326 & $33.0(27.9-39.2)$ \\
\hline PH (SPAP > 50 mmHg) & 326 & $20(6 \%)$ \\
\hline RVFAC (\%) & 342 & $40.1(36.1-44.8)$ \\
\hline RVfwLS (\%) & 342 & $24.6(20.7-27.8)$ \\
\hline RVGLS (\%) & 342 & $19.4(16.6-21.8)$ \\
\hline Peak velocity (m/s) & 366 & $3.04(2.53-3.59)$ \\
\hline Mean PG (mmHg) & 366 & $19.4(13.8-29.8)$ \\
\hline Indexed AVA $\left(\mathrm{cm}^{2} / \mathrm{m}^{2}\right)$ & 354 & $0.64(0.51-0.80)$ \\
\hline AS classification & 354 & \\
\hline HG-AS & & $44(12 \%)$ \\
\hline LFLG-AS with preserved LVEF & & $24(7 \%)$ \\
\hline LFLG-AS with reduced LVEF & & $21(6 \%)$ \\
\hline NFLG-AS & & $108(30 \%)$ \\
\hline Mild to moderate AS & & $158(45 \%)$ \\
\hline SVi $\left(\mathrm{mL} / \mathrm{m}^{2}\right)$ & 355 & $42.5(37.0-49.1)$ \\
\hline 3D LVEDVI $\left(\mathrm{mL} / \mathrm{m}^{2}\right)$ & 355 & $83.3(72.1-97.3)$ \\
\hline 3D LVESVI $\left(\mathrm{mL} / \mathrm{m}^{2}\right)$ & 355 & $39.9(33.1-49.2)$ \\
\hline 3D LVEF (\%) & 355 & $52.5(47.9-55.6)$ \\
\hline 3D LVMI $\left(\mathrm{g} / \mathrm{m}^{2}\right)$ & 355 & $98.8(83.9-113.6)$ \\
\hline 3D LAVIx (mL/m²) & 345 & $50.1(39.7-61.3)$ \\
\hline 3D LAVIn $\left(\mathrm{mL} / \mathrm{m}^{2}\right)$ & 345 & $26.9(21.1-36.1)$ \\
\hline 3D RVEDVI (mL/m²) & 355 & $55.9(48.4-66.4)$ \\
\hline 3D RVESVI $\left(\mathrm{mL} / \mathrm{m}^{2}\right)$ & 355 & $29.4(24.2-34.1)$ \\
\hline 3D RVEF (1\%) & 355 & $48.0(43.7-52.7)$ \\
\hline
\end{tabular}

Data are shown as medians (25th to 75th percentiles) or $n$ (\%).

$3 D$, three-dimensional; AVA, aortic valve area; $L A E F$, left atrial emptying fraction: LAVIn, minimum left atrial volume index; LAVIx, maximum left atrial volume index; $L V E D V I$, left ventricular end-diastolic volume index; $L V E F$, left ventricular ejection fraction; LVESVI, left ventricular end-systolic volume index; LVMI, left ventricular mass index; MR, mitral regurgitation; $P G$, pressure gradient; $P H$, pulmonary hypertension; RVEDVI, right ventricular end-diastolic volume index; RVEF, right ventricular ejection fraction; RVESVI, right ventricular end-systolic volume index; RVFAC, right ventricular fractional area change; RVfwLS, right ventricular free-wall longitudinal strain; RVGLS, right ventricular global longitudinal strain; SPAP, systolic pulmonary artery pressure; SVi, stroke volume index; TR, tricuspid regurgitation.

LVMI, SVi, LAVIx, LAVIn, peak velocity, mean PG, iAVA, E wave, E/e', RVEDVI, RVESVI, and RVEF. CART first selected RVEF (with a cut-off of $41 \%$ ), followed by LVEF (with a cut-off of 39\%), Charlson's cormorbidity index, LAVIn, and mean PG (Figure 3). Generated Kaplan-Meier curves indicated that only $4 \%$ of patients (9/212) having more than cut-off values of LVEF, RVEF, and Charlson's comorbidity index $\leq 6$ developed cardiac events. Corresponding values of patients with $\leq 41 \%$ of RVEF and $\leq 39 \%$ of LVEF were $58 \%(30 / 52)$ and $43 \%$ (6/14), respectively.

For the Kaplan-Meier analysis, we divided patients into four groups according to cut-off values of LVEF (50\%) and RVEF (45\%) (Figure 4). There was a significant difference between the 
TABLE 3 | Univariate Cox regression analyses of predictors of cardiac events.

\begin{tabular}{|c|c|c|c|c|}
\hline & HR & $95 \% \mathrm{Cl}$ & Z-score & $P$-value \\
\hline Age (per 1 y.o increase) & 1.025 & $0.994-1.058$ & 1.569 & 0.117 \\
\hline Sex (Male) & 1.237 & $0.735-2.083$ & 0.801 & 0.423 \\
\hline BMI (per 1 kg/m² increase) & 0.956 & $0.888-1.030$ & -1.181 & 0.238 \\
\hline BSA (per $1 \mathrm{~m}^{2}$ increase) & 0.738 & $0.196-2.787$ & -0.448 & 0.654 \\
\hline SBP (per 1 mmHg increase) & 0.993 & $0.982-1.004$ & -1.318 & 0.188 \\
\hline DBP (per 1mmHg increase) & 0.996 & $0.977-1.015$ & -0.419 & 0.675 \\
\hline Heat Rate (per 1 bpm increase) & 1.030 & $1.010-1.051$ & 2.899 & 0.004 \\
\hline E-wave (per $1 \mathrm{~cm} / \mathrm{s}$ increase) & 1.010 & $1.003-1.017$ & 2.809 & 0.005 \\
\hline E/A (per 1-unit increase) & 1.298 & $0.808-2.087$ & 1.078 & 0.281 \\
\hline E/e' (per 1-unit increase) & 1.031 & $1.013-1.049$ & 3.349 & $<0.001$ \\
\hline MR (moderate or severe) & 2.303 & $0.557-0.529$ & 1.152 & 0.250 \\
\hline TR (moderate or severe) & 2.301 & $0.700-7.569$ & 1.372 & 0.170 \\
\hline SPAP (per 1 mmHg increase) & 1.015 & $0.989-1.042$ & 1.117 & 0.264 \\
\hline $\mathrm{PH}$ (yes) & 2.874 & $1.222-6.757$ & 2.420 & 0.016 \\
\hline RVFAC (per 1\% increase) & 0.916 & $0.884-0.949$ & -4.866 & $<0.001$ \\
\hline RVfwLS (per 1\% increase) & 0.887 & $0.841-0.936$ & -4.399 & $<0.001$ \\
\hline RVGLS (per 1\% increase) & 0.820 & $0.765-0.880$ & -5.535 & $<0.001$ \\
\hline Peak velocity (per 1 m/s increase) & 1.283 & $0.911-1.081$ & 1.427 & 0.153 \\
\hline Mean PG (per 1 mmHg increase) & 1.020 & $1.002-1.039$ & 2.163 & 0.030 \\
\hline Indexed AVA (per $1 \mathrm{~cm}^{2} / \mathrm{m}^{2}$ increase) & 0.065 & $0.013-0.317$ & -3.384 & $<0.001$ \\
\hline SVi (per $1 \mathrm{~mL} / \mathrm{m}^{2}$ increase) & 0.945 & $0.916-0.975$ & -3.598 & $<0.001$ \\
\hline AVR (yes) & 0.539 & $0.272-1.335$ & -1.335 & 0.182 \\
\hline Charlson's index (per 1-point increase) & 1.296 & $1.137-1.487$ & 3.876 & $<0.001$ \\
\hline 3D LVEDVI (per $1 \mathrm{~mL} / \mathrm{m}^{2}$ increase) & 1.006 & $0.994-1.017$ & 0.965 & 0.335 \\
\hline 3D LVESVI (per 1 mL/m² increase) & 1.027 & $1.014-1.039$ & 4.215 & $<0.001$ \\
\hline 3D LVEF (per 1\% increase) & 0.894 & $0.868-0.920$ & -7.559 & $<0.001$ \\
\hline 3D LVMI (per $1 \mathrm{~g} / \mathrm{m}^{2}$ increase) & 1.012 & $1.004-1.020$ & 2.979 & 0.003 \\
\hline 3D LAVIx (per $1 \mathrm{~mL} / \mathrm{m}^{2}$ increase) & 1.023 & $1.011-1.036$ & 3.812 & $<0.001$ \\
\hline 3D LAVIn (per $1 \mathrm{~mL} / \mathrm{m}^{2}$ increase) & 1.034 & $1.022-1.046$ & 5.508 & $<0.001$ \\
\hline 3D LAEF (per 1\% increase) & 0.941 & $0.921-0.960$ & -5.790 & $<0.001$ \\
\hline 3D RVEDVI (per $1 \mathrm{~mL} / \mathrm{m}^{2}$ increase) & 1.017 & $1.003-1.032$ & 2.435 & 0.015 \\
\hline 3D RVESVI (per $1 \mathrm{~mL} / \mathrm{m}^{2}$ increase) & 1.060 & $1.041-1.079$ & 6.437 & $<0.001$ \\
\hline 3D RVEF (per 1\% increase) & 0.891 & $0.864-0.917$ & -7.669 & $<0.001$ \\
\hline
\end{tabular}

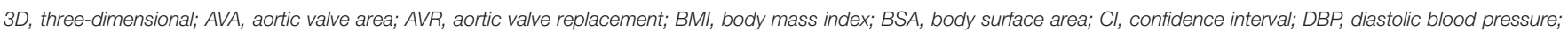

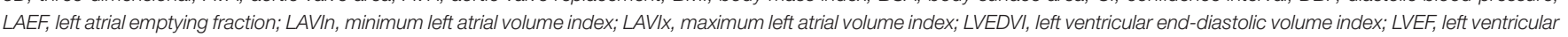

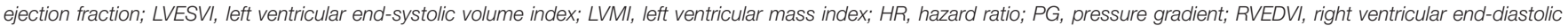

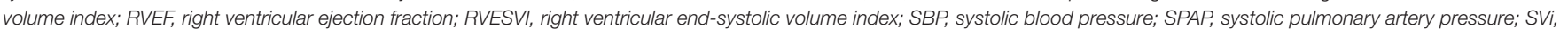
stroke volume index.

group of patients who had preserved LVEF with impaired RVEF and the group of patients with both EFs preserved $(p<0.001)$.

\section{RVEF and Conventional 2D RV Parameters}

RVGLS was significantly associated with cardiac events even after being adjusted for AVR, Charlson's comorbidity index, LVEF, and either mean PG, iAVA, E/e, LAVIn, or SVi (Supplementary Tables S1-S3). RVfwLS had no significant association with outcome when LAVIn was included in the multivariate model. However, when we added RVEF to the multivariate model, the prognostic significance of all of three 2D RV parameters disappeared (Supplementary Table S4). To determine the independent and incremental prognostic value of RVEF, we generated a nested regression model, sequentially adding iAVA, LVEF, conventional 2D RV parameters (either FAC of RVGLS), and RVEF. Chi-square values increased in stepwise fashion upon adding each parameter. Addition of RVEF to the model including iAVA, LVEF and RVFAC further increased the chi-square value from 57.7 to $71.7(p<0.001)$, whereas adding it to the model containing iAVA, LVEF and RVGLS increased it from 61.5 to 72.7 ( $p<0.001)$, respectively (Supplementary Figure S1).

\section{Patients With Preserved LVEF}

For sensitivity analysis, we performed subgroup analyses in a subset of patients who had $\geq 50 \%$ of LVEF. Among 248 patients 
TABLE 4 | Multivariate Cox regression analyses after adjusting Charlson index and AVR as time-dependent covariates.

\begin{tabular}{|c|c|c|c|c|c|c|c|c|c|c|}
\hline & \multicolumn{2}{|c|}{ Mean PG model } & \multicolumn{2}{|l|}{ iAVA model } & \multicolumn{2}{|l|}{ SVi model } & \multicolumn{2}{|l|}{ E/e' model } & \multicolumn{2}{|l|}{ LAVIn model } \\
\hline & HR (95\% Cl) & $P$-value & HR (95\% Cl) & $P$-value & HR (95\% Cl) & $P$-value & HR (95\% Cl) & $P$-value & HR (95\% Cl) & $P$-value \\
\hline LVEF & $0.933(0.900-0.968)$ & $<0.001$ & $0.944(0.910-0.978)$ & 0.002 & 0.945 (0.908-0.983) & 0.005 & $0.951(0.916-0.988)$ & 0.010 & $0.954(0.917-0.993)$ & 0.021 \\
\hline RVEF & $0.918(0.887-0.949)$ & $<0.001$ & $0.924(0.893-0.956)$ & $<0.001$ & $0.918(0.884-0.954)$ & $<0.001$ & $0.913(0.881-0.947)$ & $<0.001$ & $0.926(0.892-0.961)$ & $<0.001$ \\
\hline Mean PG & $1.038(1.020-1.055)$ & $<0.001$ & & & & & & & & \\
\hline iAVA & & & $0.073(0.015-0.357)$ & 0.001 & & & & & & \\
\hline SVi & & & & & 0.999 (0.971-1.029) & 0.981 & & & & \\
\hline E/e' & & & & & & & 1.035 (1.009-1.062) & 0.009 & & \\
\hline LAVIn & & & & & & & & & $1.024(1.008-1.039)$ & 0.002 \\
\hline
\end{tabular}

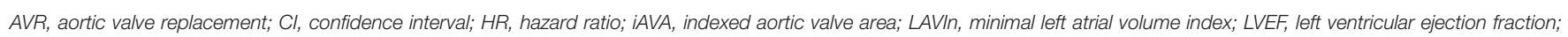
RVEF, right ventricular ejection fraction; SVi, stroke volume index.

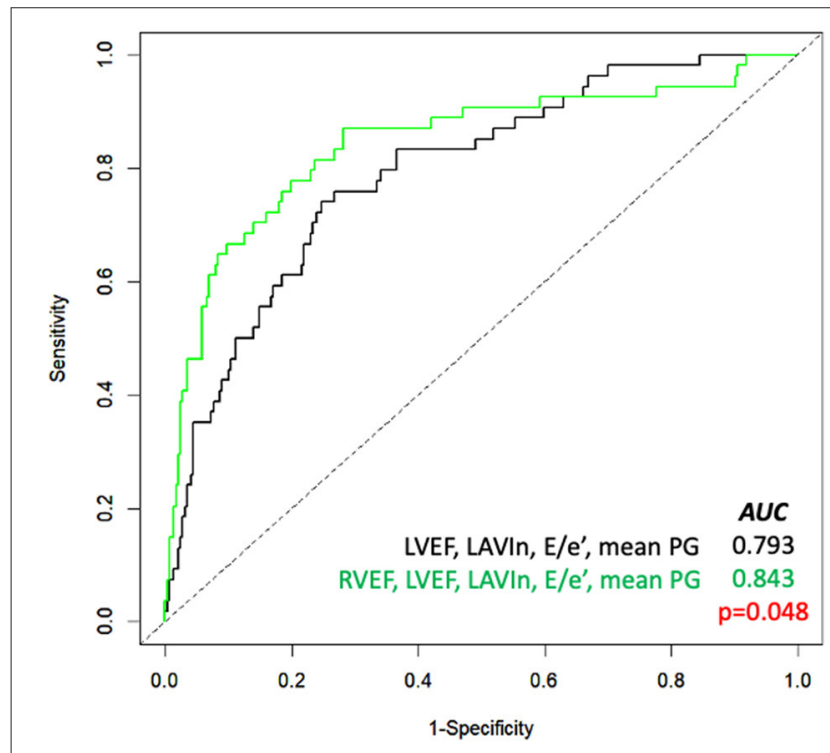

FIGURE 2 | Receiver-operating characteristic curve analysis for prediction of cardiac events. Comparison of the area under the curve (AUC) of several parameters (black line) and with RVEF added (green line). LAVIn, minimum left atrial volume index; LVEF, left ventricular ejection fraction; PG, pressure gradient; RVEF, right ventricular ejection fraction.

with $\mathrm{LVEF} \geq 50 \%, 23$ patients developed cardiac events during a median of 32 months follow-up. Multivariate Cox analysis, revealed that RVEF was a significant marker even after adjusting for LVEF $(p<0.001)$. Supplementary Figure S2 shows the results of the CART analysis. As with the results for all patients, CART selected RVEF first.

\section{Patients With}

\section{"More-Than-Moderate-to-Severe AS"}

Among 196 "more-than-moderate-to-severe" AS patients, 40 developed cardiac events during a median of 30 months follow-up. Univariate Cox regression analysis indicated that RVEF and conventional 2DE RV parameters were significantly associated with cardiac events (Supplementary Table S5). The results of multivariate Cox regression analyses revealed that RVEF was significantly associated with cardiac events, even after adjusting for AVR, Charlson index, and LVEF / iAVA/ LVMI /E/e' /LAVIn (Supplementary Table S6). We constructed a nested regression model incorporating iAVA, LVEF, RVFAC or RVLS, and RVEF in stepwise manner to determine the independent and incremental prognostic value of RVEF. RVEF had a significant incremental value over iAVA, LVEF, and RVFAC (Supplementary Figure S3, left panel) and over iAVA, LVEF, and RVGLS (Supplementary Figure S3, right panel). Supplementary Figure S4 shows the CART analysis indicating that LVEF was selected first with a cut-off value of $47 \%$, followed by RVESVI with a cut-off value of $39 \mathrm{~mL} / \mathrm{m}^{2}$.

\section{Patients With "Less-Than-Moderate-to-Severe AS"}

Among 158 "less-than-moderate-to-severe" AS patients, 16 developed cardiac events during a median of 28 months followup. Univariate Cox regression analysis revealed that RVEF and RVGLS were significantly associated with cardiac events (Supplementary Table S7). CART analysis selected RVEF at first with a cut-off value of $42 \%$ (Supplementary Figure S5).

\section{Factors Associated With Reduced RVEF}

We performed logistic regression analysis to determine which parameter was associated with reduced RVEF, which was defined as RVEF $<40 \%$. Univariate logistic regression analysis revealed that heart rate, E/e', iAVA, SVi, LVEF, LAVIn, coronary artery disease (CAD), chronic kidney disease (CKD), and atrial fibrillation (AF) were significantly associated with reduced RVEF. Multivariate logistic regression analyses revealed that iAVA, SVi, LVEF, and LAVIn were significantly associated with reduced RVEF, even after adjusting for CAD, CKD, and AF (Supplementary Table S8).

\section{DISCUSSION}

To the best of our knowledge, this is the first study to demonstrate the prognostic utility of 3DE determined RVEF in patients with asymptomatic AS. 


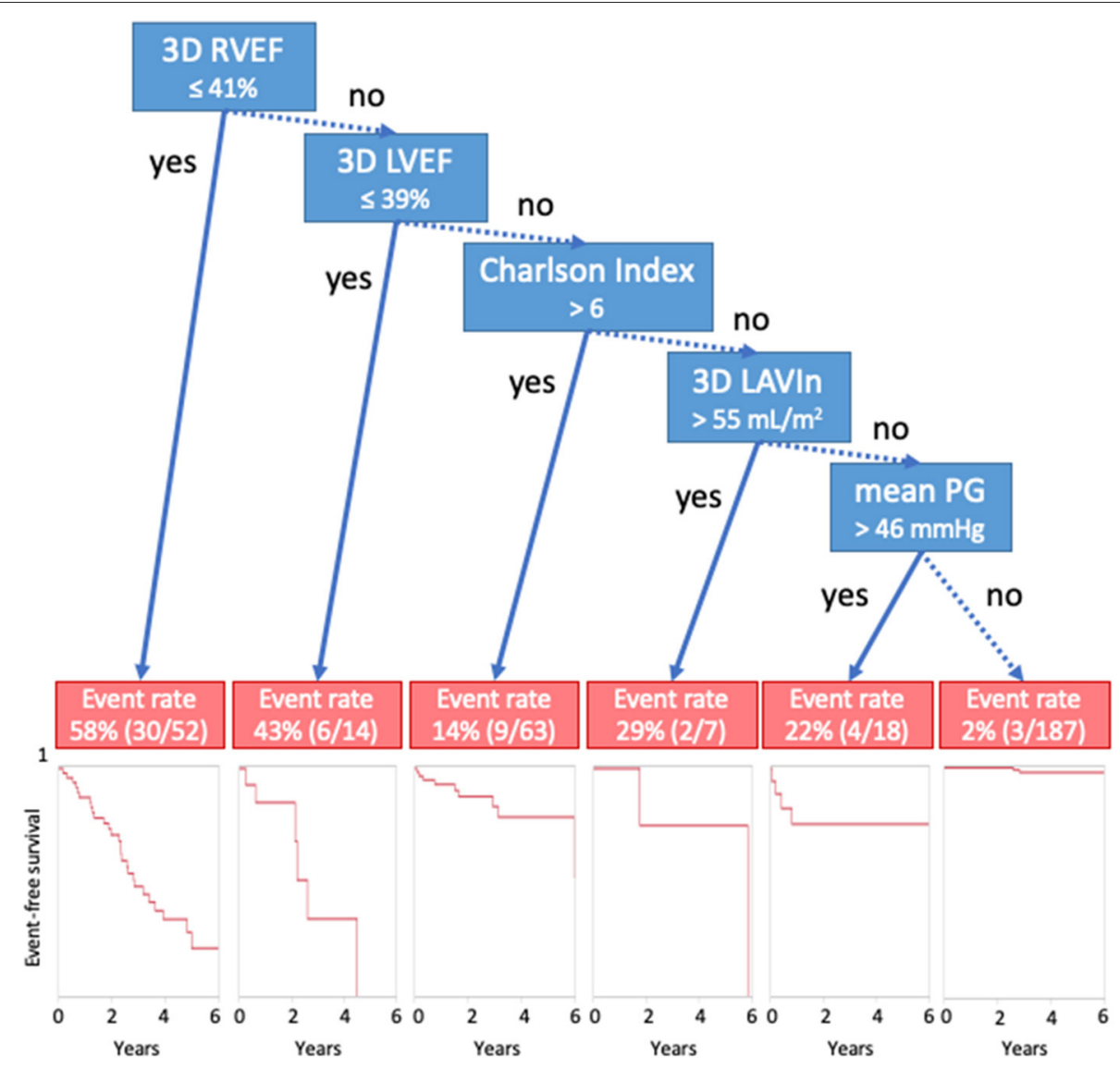

FIGURE 3 | Classification and regression-tree analysis for cardiac events. Blue boxes denote cut-offs, and red boxes describe event rates. 3D, three-dimensional; AVR, aortic valve replacement; LAVIn, minimum left atrial volume index; LVEF, left ventricular ejection fraction; PG, pressure gradient; RVEF, right ventricular ejection fraction.

\section{Previous Studies}

Indications and best timing of AVR for patients with asymptomatic AS are not clearly defined. Some studies suggest that early elective AVR improves the prognosis of patients with asymptomatic severe AS (21). Genereux et al. proposed the "cardiac damage" concept, which claims that damage to each heart chamber regulates the prognosis of patients with severe AS who received AVR, and their results suggest that when RV damage has occurred, it is too late to intervene (12). Moreover, Tastes et al. reported that the cardiac damage system has significant prognostic utility for patients with moderate to severe asymptomatic AS, independent of AVR (13). Because of its reduced invasiveness and lower perioperative risk, the advent of transcatheter AVR could increase the frequency of valve replacement in patients who are at high risk for surgical AVR. For this reason, pre-operative risk stratification of patients with asymptomatic AS is vital for optimal use of health resources.

Some reports have explained the prognostic utility of RV functional parameters in AS. Galli et al. reported the prognostic importance of RV function among 200 patients with severe AS (14). The subjects were predominantly asymptomatic (87\%). They found that $24 \%$ of severe AS patients had impaired RV function, and biventricular dysfunction was a strong prognostic indicator of future cardiac events. Zilberszac et al. reported the prognostic value of RV dysfunction in 76 patients with lowflow, low-gradient severe AS (22). Only seven patients were asymptomatic. RV dysfunction was assessed by TAPSE, the systolic lateral tricuspid annulus velocity or $2 \mathrm{D}$ RV free-wall strain. RV dysfunction was a significant prognostic marker for overall survival in univariate Cox proportional hazard analysis. However, this utility was no longer significant after adjusting EuroSCORE II. Those two papers used only RV parameters that represent longitudinal motion. This limitation may explain why in those studies, RV parameters were not such strong markers compared with other factors, because the RV moves threedimensionally, and regional and longitudinal motion may not represent global RV function in some cases (23).

\section{Current Study}

We found that 3D RVEF had significant utility in predicting future cardiac events, even after adjusting for LVEF, AS severity, LV diastolic function, AVR, and comorbidities. Statistical analysis revealed that RVEF had incremental value over comorbidities, left heart parameters, and AS severity to predict cardiac events. 


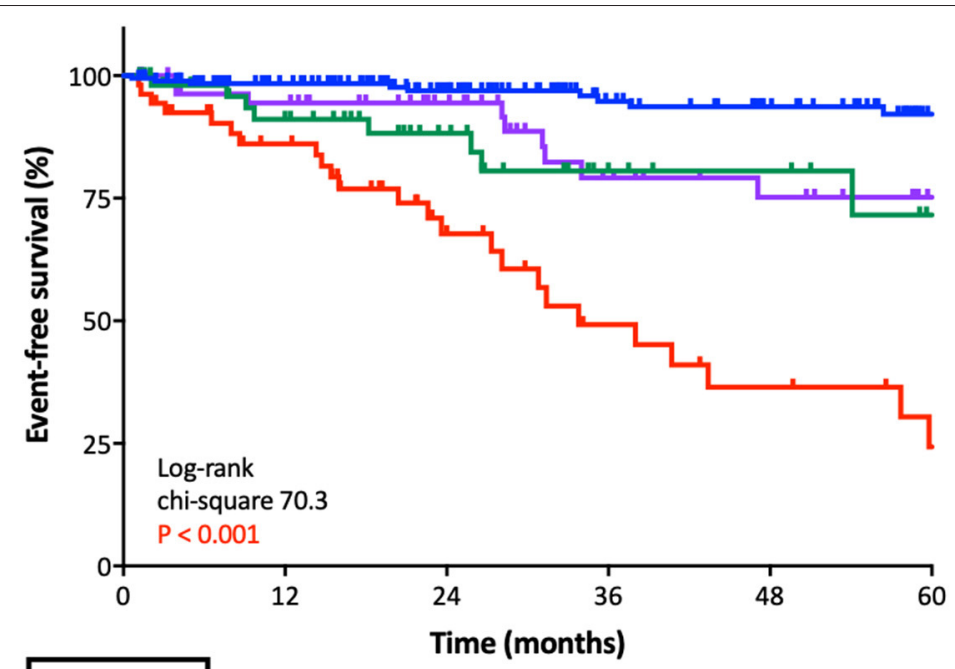

Number at risk

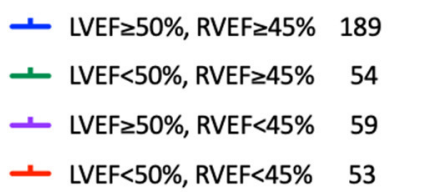

$\begin{array}{ll}158 & 122 \\ 39 & 26 \\ 51 & 40 \\ 40 & 21\end{array}$

88
14
25
13

$\begin{array}{cc}74 & 52 \\ 12 & 7 \\ 20 & 13 \\ 9 & 5\end{array}$

FIGURE 4 | Kaplan-Meier survival analysis for cardiac events based upon RVEF and LVEF. Kaplan-Meier analysis for cardiac events, according to group, as classified by lower limits of normal LVEF and RVEF. LVEF, left ventricular ejection fraction; RVEF, right ventricular ejection fraction.

Kaplan-Meier analysis indicated that both LVEF and RVEF were useful for risk stratification of patients with asymptomatic AS.

In our cohort, $73 \%$ of asymptomatic AS patients had some non-cardiac comorbidities. Not only AS severity and left heart function, but also other comorbidities that develop RV volume or pressure overload, such as pulmonary disease and CKD can affect RV function. Thus, RV function may represent status of the whole heart. In fact, reduced RVEF was significantly associated with echocardiographic parameters, CKD, CAD, and atrial fibrillation. We cannot determine the causal relationship because of the cross sectional nature of the study; however, optimal medical therapy or intervention targeting HF, ischemia, and AF have potential to improve management of AS patients with reduced RVEF.

Our results are consistent with previous publications. Previously, we determined the prognostic utility of 3D RVEF in 446 patients with diverse backgrounds (18). 3D RVEF had an incremental value over $3 \mathrm{D} \mathrm{LVEF}, \mathrm{E} / \mathrm{e}$, and $\mathrm{CKD}$ to predict future adverse events. CART analysis selected RVEF first with a cut-off of $41 \%$. The value was equivalent to observed value in this study (Figure 3). Muraru et al. also reported the prognostic utility of 3D RVEF in patients with various cardiac diseases (24). They stressed that 3D RVEF showed higher predictive power for cardiac events than TAPSE or RVFAC, which was in agreement with our results. Kaplan-Meier analysis indicated that patients with RVEF $<40 \%$ showed a high incidence of cardiac death, cardiac events, and allcause mortality. Those results are consistent with our RVEF cutoff values derived from CART analysis. Unlike those two previous studies, our intent was to determine prognostic value of RVEF in a specific group of patients, asymptomatic AS, and we clearly showed its prognostic utility.

In a subset of patients with "more-than-moderate-tosevere" AS, CART analysis selected LVEF first. The current AHA/ACC guideline (3) recommends aortic valve surgery as class I indication for asymptomatic severe AS patients with LVEF $<50 \%$. Therefore, we believe that assessing LVEF at first is reasonable in asymptomatic severe AS. CART secondly selected RVESVI. However, we must note that 7 of 28 more-than-moderate-to-severe AS patients with $\mathrm{LVEF} \geq 50 \%$, who also had RVEF $<45 \%$ developed cardiac events. Of those 7 patients who had cardiac events, two developed heart failure after AVR. Thus, when we manage asymptomatic severe AS patients, we should assess LV function for indications of AVR first. However, we should carefully manage patients with preserved LVEF, but impaired RVEF. On the other hand, for patients with asymptomatic mild/moderate AS, the prognostic importance of RVEF may be greater than that of left heart function and valve condition.

\section{Clinical Implications}

Assessment of RVEF using 3DE provides robust information for predicting future outcomes in patients with asymptomatic AS. Overall, 3D RVEF was significantly associated with cardiac events regardless of AS severity or left chamber function. 3DE determined that RVEF is a much more useful parameter than 2DE-derived RV parameters for predicting future outcome. Thus, combined 3DE assessment of left and right chamber function 
might be the best way for appropriate management of patients with asymptomatic AS.

\section{LIMITATIONS}

This study has several limitations. First, it includes not only severe AS patients, but also mild to moderate AS patients. However, because AS is a progressive disease, predicting future outcomes of patients with mild to moderate AS using baseline echocardiography is essential for appropriate management. In fact, RV systolic functions were significant prognostic parameters, independent of AS severity. Second, 3D LAV analysis was based on manual tracing, even though $3 \mathrm{D} L V$ and $\mathrm{RV}$ analyses were performed using semi-automated 3D speckle tracking software. This difference could underrepresent the prognostic value of LAVs in this study, and further studies using 3D LA speckle tracking analysis are required. Third, we did not include $3 \mathrm{D}$ global strain values. It is crucial to determine whether $3 \mathrm{D}$ global strain values are superior to EFs and volumetric parameters. However, there are few commercial software packages that can incorporate 3D RV and LA strains. Fourth, although we demonstrated that 3D RVEF is better than $2 \mathrm{D}$ RV parameters, its accuracy and reliability depend on the expertise of the examiner. 3DE data acquisition was not always possible in every AS patient. In such situations, 2D RVFAC and RVGLS are a potential alternative. Fifth, although significant tricuspid regurgitation (TR) and mitral regurgitation (MR) have potential to affect the prognosis of patients with AS, there were not significant association with cardiac events in this study. This may be due to the small sample size in more than moderate TR and MR. Finally, this was a single-center retrospective study. To validate our concepts, a multi-center prospective study is required.

\section{REFERENCES}

1. Benjamin EJ, Virani SS, Callaway CW, Chamberlain AM, Chang AR, Cheng S, et al. Heart disease and stroke statistics-2018 update: a report from the American Heart Association. Circulation. (2018) 137:e67-492. doi: 10.1161/CIR.0000000000000558

2. Goldsweig AM, Tak HJ, Chen LW, Aronow HD, Shah B, Kolte DS, et al. The evolving management of aortic valve disease: 5-year trends in SAVR, TAVR, and medical therapy. Am J Cardiol. (2019) 124:763-71. doi: 10.1016/j.amjcard.2019.05.044

3. Nishimura RA, Otto CM, Bonow RO, Carabello BA, Erwin JP, Fleisher LA, et al. 2017 AHA/ACC focused update of the 2014 AHA/ACC guideline for the management of patients with valvular heart disease: a report of the American College of Cardiology/American Heart Association Task Force on Clinical Practice Guidelines. Circulation. (2017) 135:e1159-95. doi: 10.1161/CIR.0000000000000503

4. Pai RG, Kapoor N, Bansal RC, Varadarajan P. Malignant natural history of asymptomatic severe aortic stenosis: benefit of aortic valve replacement. Ann Thorac Surg. (2006) 82:2116-22. doi: 10.1016/j.athoracsur.2006.07.043

5. Pellikka PA, Nishimura RA, Bailey KR, Tajik AJ. The natural history of adults with asymptomatic, hemodynamically significant aortic stenosis. J Am Coll Cardiol. (1990) 15:1012-7. doi: 10.1016/0735-1097(90)90234-G

6. Pellikka PA, Sarano ME, Nishimura RA, Malouf JF, Bailey KR, Scott CG, et al. Outcome of 622 adults with asymptomatic, hemodynamically significant

\section{CONCLUSIONS}

3D RVEF had significant prognostic value, even after adjusting 3D left heart parameters and comorbidities in patients with asymptomatic AS. A cut-off value of RVEF $\leq 40 \%$ should be considered for better management in asymptomatic AS patients.

\section{DATA AVAILABILITY STATEMENT}

The raw data supporting the conclusions of this article will be made available by the authors, without undue reservation.

\section{ETHICS STATEMENT}

The studies involving human participants were reviewed and approved by the Ethics Committee at the University of Occupational and Environmental Health. Written informed consent for participation was not required for this study in accordance with the national legislation and the institutional requirements.

\section{AUTHOR CONTRIBUTIONS}

YN: data curation, data analysis, and writing-draft. TK: data curation. MT: conceptualization, methodology, supervision, writing-review, and editing. All authors contributed to the article and approved the submitted version.

\section{SUPPLEMENTARY MATERIAL}

The Supplementary Material for this article can be found online at: https://www.frontiersin.org/articles/10.3389/fcvm. 2021.795016/full\#supplementary-material

aortic stenosis during prolonged follow-up. Circulation. (2005) 111:3290-5. doi: 10.1161/CIRCULATIONAHA.104.495903

7. Rosenhek R, Binder T, Porenta G, Lang I, Christ G, Schemper M, et al. Predictors of outcome in severe, asymptomatic aortic stenosis. $N$ Engl J Med. (2000) 343:611-7. doi: 10.1056/NEJM2000083134 30903

8. Dal-Bianco JP, Khandheria BK, Mookadam F, Gentile F, Sengupta PP. Management of asymptomatic severe aortic stenosis. J Am Coll Cardiol. (2008) 52:1279-92. doi: 10.1016/j.jacc.2008.07.020

9. Lancellotti P, Magne J, Dulgheru R, Clavel MA, Donal E, Vannan $\mathrm{MA}$, et al. Outcomes of patients with asymptomatic aortic stenosis followed up in heart valve clinics. JAMA Cardiol. (2018) 3:1060-8. doi: 10.1001/jamacardio.2018.3152

10. Otto CM. Valvular aortic stenosis: disease severity and timing of intervention. J Am Coll Cardiol. (2006) 47:2141-51. doi: 10.1016/j.jacc.2006.03.002

11. Purmah Y, Lei LY, Dykstra S, Mikami Y, Cornhill A, Satriano A, et al. Right ventricular ejection fraction for the prediction of major adverse cardiovascular and heart failure-related events: a cardiac MRI based study of 7131 patients with known or suspected cardiovascular disease. Circ Cardiovasc Imaging. (2021) 14:3. doi: 10.1161/CIRCIMAGING.120.0 11337

12. Genereux P, Pibarot P, Redfors B, Mack MJ, Makkar RR, Jaber WA, et al. Staging classification of aortic stenosis based on the extent of cardiac damage. Eur Heart J. (2017) 38:3351-8. doi: 10.1093/eurheartj/ehx381 
13. Tastet L, Tribouilloy C, Marechaux S, Vollema EM, Delgado V, Salaun E, et al. Staging cardiac damage in patients with asymptomatic aortic valve stenosis. $J$ Am Coll Cardiol. (2019) 74:550-63. doi: 10.1016/j.jacc.2019.04.065

14. Galli E, Guirette Y, Feneon D, Daudin M, Fournet M, Leguerrier A, et al. Prevalence and prognostic value of right ventricular dysfunction in severe aortic stenosis. Eur Heart J Cardiovasc Imaging. (2015) 16:531-8. doi: 10.1093/ehjci/jeu290

15. Lang RM, Badano LP, Mor-Avi V, Afilalo J, Armstrong A, Ernande L, et al. Recommendations for cardiac chamber quantification by echocardiography in adults: an update from the American Society of Echocardiography and the European Association of Cardiovascular Imaging. J Am Soc Echocardiogr. (2015) 28:1-39. doi: 10.1016/j.echo.2014.10.003

16. Todaro MC, Carerj S, Khandheria B, Cusma-Piccione M, La Carrubba $\mathrm{S}$, Antonini-Canterin F, et al. Usefulness of atrial function for risk stratification in asymptomatic severe aortic stenosis. J Cardiol. (2016) 67:71-9. doi: $10.1016 / j . j j$ jcc.2015.04.010

17. Charlson ME, Pompei P, Ales KL, MacKenzie CR. A new method of classifying prognostic comorbidity in longitudinal studies: development and validation. J Chronic Dis. (1987) 40:373-83. doi: 10.1016/0021-9681(87)90171-8

18. Nagata Y, Wu VC, Kado Y, Otani K, Lin FC, Otsuji Y, et al. Prognostic value of right ventricular ejection fraction assessed by transthoracic 3D echocardiography. Circ Cardiovasc Imaging. (2017) 10:2. doi: 10.1161/CIRCIMAGING.116.005384

19. Iwataki M, Takeuchi M, Otani K, Kuwaki H, Haruki N, Yoshitani H, et al. Measurement of left atrial volume from transthoracic three-dimensional echocardiographic datasets using the biplane Simpson's technique. J Am Soc Echocardiogr. (2012) 25:1319-26. doi: 10.1016/j.echo.2012.08.017

20. Vahanian A, Beyersdorf F, Praz F, Milojevic M, Baldus S, Bauersachs J, et al. 2021 ESC/EACTS Guidelines for the management of valvular heart disease. Eur Heart J. (2021) 28:ehab395. doi: 10.1093/eurheartj/e hab626

21. Miyake M, Izumi C, Taniguchi T, Morimoto T, Amano M, Nishimura S, et al. Early surgery vs. surgery after watchful waiting for asymptomatic severe aortic stenosis. Circ J. (2018) 82:2663-71. doi: 10.1253/circj.CJ18-0416

22. Zilberszac R, Gleiss A, Schweitzer R, Bruno P, Andreas M, Stelzmuller M, et al. Prognostic value of right ventricular dysfunction and tricuspid regurgitation in patients with severe low-flow low-gradient aortic stenosis. Sci Rep. (2019) 9:1. doi: 10.1038/s41598-019-51166-0

23. Lakatos BK, Nabeshima Y, Tokodi M, Nagata Y, Toser Z, Otani K, et al. Importance of nonlongitudinal motion components in right ventricular function: three-dimensional echocardiographic study in healthy volunteers. J Am Soc Echocardiogr. (2020) 33:995-1005. doi: 10.1016/j.echo.2020.04.002

24. Muraru D, Badano LP, Nagata Y, Surkova E, Nabeshima Y, Genovese D, et al. Development and prognostic validation of partition values to grade right ventricular dysfunction severity using 3D echocardiography. Eur Heart J Cardiovasc Imaging. (2020) 21:10-21. doi: 10.1093/ehjci/jez233

Conflict of Interest: The authors declare that the research was conducted in the absence of any commercial or financial relationships that could be construed as a potential conflict of interest.

Publisher's Note: All claims expressed in this article are solely those of the authors and do not necessarily represent those of their affiliated organizations, or those of the publisher, the editors and the reviewers. Any product that may be evaluated in this article, or claim that may be made by its manufacturer, is not guaranteed or endorsed by the publisher.

Copyright (c) 2021 Nabeshima, Kitano and Takeuchi. This is an open-access article distributed under the terms of the Creative Commons Attribution License (CC BY). The use, distribution or reproduction in other forums is permitted, provided the original author(s) and the copyright owner(s) are credited and that the original publication in this journal is cited, in accordance with accepted academic practice. No use, distribution or reproduction is permitted which does not comply with these terms. 